\title{
Desigualdad y logro académico en Chile
}

\author{
Pablo Muñoz H. y Amaia Redondo S.
}

RESUMEN

En este trabajo se utiliza una base de datos de panel para contribuir con nueva evidencia sobre la repercusión de los determinantes socioeconómicos en los índices de logro académico en Chile. Se advierte que los condicionantes socioeconómicos tienen un efecto estadísticamente significativo y creciente de un período a otro en el nivel de logro académico. La evidencia muestra que dos individuos de distinto nivel socioeconómico (NSE), que logran igual puntaje en el Sistema de Medición de Calidad de la Educación (SIMCE) en octavo básico, presentan una brecha de más de 70 puntos promedio cuatro años después al rendir la Prueba de Selección Universitaria (PSU). Se concluye que en un contexto de elevada desigualdad de ingresos y altos retornos a la educación terciaria, los índices de logro académico activan restricciones en el acceso a la educación superior, principalmente en la población de bajo NSE, perpetuando la mala distribución de los ingresos.

PALABRAS CLAVE

CLASIFICACIÓN JEL

AUTORES
Estructura social, alumnos, rendimiento escolar, aspectos sociales, clases sociales, medición, modelos econométricos, Chile

$\mathrm{I} 22, \mathrm{I} 24, \mathrm{I} 25, \mathrm{I} 31, \mathrm{~J} 31$

Pablo Muñoz H., Departamento de Economía de la Universidad de Chile.pabmunoz@fen.uchile.cl Amaia Redondo S., Departamento de Economía de la Universidad de Chile. aredondo@fen.uchile.cl 


\section{I}

\section{Introducción}

De acuerdo con una serie de estudios preparados por agencias internacionales, tales como la Comisión Económica para América Latina y el Caribe (CEPAL), el Banco Mundial y el Banco Interamericano de Desarrollo (BID), la distribución de ingresos en América Latina se ha caracterizado por altos y persistentes niveles de desigualdad. A mediados de la década de 2000, el coeficiente de Gini era del orden de 0,53 y América Latina era más desigual que el África subsahariana, Asia Oriental y los países de altos ingresos en un $18 \%$, un $36 \%$ y un $65 \%$, respectivamente.

En Declining Latin America Inequality: Market Forces or State Action, Luis López-Calva y Nora Lustig (2010) han mostrado que la desigualdad en la región ha disminuido a partir del año 2000. En México, la Argentina, el Perú y el Brasil dos son los principales factores que explican la reducción de la desigualdad: la disminución de la brecha de ingresos entre trabajadores calificados y no calificados, y el aumento de las transferencias del gobierno hacia los sectores más vulnerables. A pesar de ello, no es posible proyectar una aminoración continua de la desigualdad, pues la población de menores ingresos que ha logrado acceder a la educación básica y secundaria enfrenta aún varias barreras de acceso a la educación terciaria ${ }^{1}$.

Dentro de la región, Chile ha presentado tasas de crecimiento sobresalientes durante las últimas décadas y junto con ello la pobreza se ha reducido. Sin embargo, se ha mostrado empíricamente que en condiciones de alto crecimiento económico, la disminución de la pobreza y las variaciones de la desigualdad de ingresos no necesariamente se correlacionan entre sí2. En este sentido, Chile es un país bastante desigual, su índice de Gini es el más alto dentro del conjunto de países miembros de la Organización de Cooperación y Desarrollo Económicos (OCDE), y la pobreza relativa es también elevada (aproximadamente 1 de cada 5 personas se encuentra en esta condición). Tradicionalmente, como posible explicación de la desigualdad en los ingresos, se señalan las diferencias en educación que se observan

\footnotetext{
${ }^{1}$ Luis López-Calva y Nora Lustig (2010) señalan que estas barreras dicen relación básicamente con la baja calidad de la educación primaria y secundaria en América Latina.

2 Véase Contreras (1996).
}

entre los integrantes de la fuerza laboral ${ }^{3}$. Es decir, quienes acceden a la educación superior capturan mayores retornos, puesto que el mercado laboral chileno demanda más trabajo calificado que el actualmente disponible, lo que no ocurre con la mano de obra no calificada.

A principios de 1980, se introdujo en Chile un conjunto de reformas en el ámbito educacional. Se implementó un sistema de comprobantes o cupones (vouchers) bajo el cual las escuelas con financiamiento público (administradas por las municipalidades) y las escuelas privadas subvencionadas reciben un subsidio directo por cada alumno. El principal mecanismo para garantizar la calidad de la educación en Chile es la libre entrada y la competencia entre escuelas, con escasa intervención estatal.

A consecuencia de esta reforma, comenzó un masivo ingreso de escuelas privadas subvencionadas cuya participación en el total de matrículas aumentó del $30 \%$ en 1986 al $48 \%$ en 2008 , mientras que el porcentaje de estudiantes que asistía a escuelas privadas pagadas (7\%) no presentó mayor variación. Por lo general, los estudiantes de las escuelas privadas pagadas provienen de familias con alto nivel socioeconómico (NSE), no así los estudiantes de las escuelas municipales quienes pertenecen sobre todo a los dos primeros quintiles de ingreso. Por su parte, quienes asisten a las escuelas subvencionadas provienen de familias con distintos $\mathrm{NSE}^{4}$, principalmente de los quintiles 3 y 4 , aunque cabe señalar que el nivel de ingreso de estos quintiles es muy inferior al de la clase media de los países de la ocDE (la razón de ingreso promedio ajustado por hogar de Chile con respecto a la OCDE es de $38 \%)^{5}$.

Durante las últimas décadas, Chile ha mejorado en cobertura educacional, no así en calidad y equidad. El proceso de reforma ha generado una mayor segregación socioeconómica y cultural ${ }^{6}$, mientras que el sistema de "cupones" no ha fomentado una competencia capaz de garantizar una mayor eficiencia, sino que ha privilegiado

\footnotetext{
3 Véase Contreras y Gallegos (2011).

${ }^{4}$ Esto se debe a que en Chile opera un sistema de financiamiento compartido, es decir, el Estado subsidia en parte a los establecimientos y las familias también realizan un copago.

5 De acuerdo con el Índice para una vida mejor (Better Life Index) de la OCDE (2012).

6 Véase Valenzuela (2006).
} 
un equilibrio en que los colegios aprovechan la posibilidad de seleccionar a objeto de minimizar costos, obteniendo calidad a costa de estratificación 7 . Ha tenido lugar un proceso de descreme, tal como lo plantean Epple y Romano (1998), restringiendo con ello la calidad de la educación a la que acceden los más pobres ${ }^{8}$. En la práctica, las escuelas privadas más onerosas cuentan con recursos por alumno significativamente más abundantes que las escuelas municipales, o que aquellas escuelas privadas subvencionadas ubicadas en las áreas más vulnerables. En diversas investigaciones se revela que el antecedente socioeconómico del alumnado es uno de los determinantes más importantes del aprendizaje, mientras que el efecto del estrato socioeconómico de otros alumnos en la misma clase puede llegar a ser incluso más pronunciado (OCDE, 2007). Por lo tanto, enseñar a estudiantes en riesgo social es difícil y por ende costoso, más aún si se concentran en una misma escuela. Otros datos ponen de manifiesto que los padres cuentan con poca información sobre la calidad de la escuela (Elacqua y Fábrega, 2004), y el acceso y uso de esta información depende del estrato socioeconómico. Además, los padres de menores recursos otorgan especial valor a la distancia entre la escuela y su hogar, independientemente de la calidad de la escuela (Chumacero, Gómez Caorsi y Paredes, 2008; Gallego y Hernando, 2009).

Lo anterior reduce los incentivos de las escuelas a elevar su calidad para atraer estudiantes. Y precisamente es en materia de calidad que Chile presenta aún grandes desafíos, ya que si bien sus resultados en el Programa Internacional de Evaluación de Estudiantes (PISA) mejoraron considerablemente entre 2000 y 2009, las calificaciones de los jóvenes de 15 años de edad en ciencias, lectura y matemáticas aún se ubican muy por debajo del promedio de la OCDE, incluso después de ajustarlas por nivel de ingresos. Cabe señalar que en Chile los resultados en la prueba se desploman, dependiendo del tipo de escuela, en directa relación con el origen socioeconómico de los estudiantes.

Ahora bien, con respecto al sistema de educación universitaria en Chile, a partir de la reforma a la educación superior de 1981 este se puede dividir en dos grandes grupos. Por una parte se encuentran las universidades privadas y por otra aquellas agrupadas en el Consejo de Rectores de las Universidades Chilenas (CRUCH). Las universidades del CRUCH concentran el mayor porcentaje de matriculados en la educación superior y además estas

\footnotetext{
7 Véase Contreras, Sepúlveda y Bustos (2007).

8 Véase Hsieh y Urquiola (2006).
}

instituciones seleccionan a los alumnos que ingresan a sus carreras utilizando un sistema común de admisión ${ }^{9}$. Desde el año 2003, el sistema de admisión del CRUCH consiste en emplear como factores de discriminación los resultados de las distintas pruebas de selección universitaria (Prueba de Selección Universitaria (PSU) en lenguaje, matemáticas, ciencia e historia), en conjunto con el promedio de notas de enseñanza media (NEM) de los postulantes ${ }^{10}$. A su vez, en el proceso de admisión de 2013 se consideró la inclusión de un instrumento adicional de medición, que consiste en ordenar a los estudiantes según su desempeño académico en la enseñanza media y premiar su posición relativa dentro de cada establecimiento educacional ${ }^{11}$. Con esta medida se atiende al hecho de que varios autores han mostrado que índices tales como la habilidad relativa, además de generar una menor exclusión socioeconómica, son buenos predictores del rendimiento en la educación superior, incluso controlando por los puntajes obtenidos en la PSU ${ }^{12}$.

Dependiendo de cada carrera y universidad, los factores señalados se ponderan de diversas maneras con el objeto de predecir el éxito académico de los alumnos durante sus estudios universitarios. En este contexto, quienes obtienen puntajes más altos tienen mayores posibilidades de elegir entre universidades y carreras, y con ello mejor acceso a los altos retornos que algunas de estas suponen.

Se ha mostrado que en Chile los resultados del Sistema de Medición de Calidad de la Educación (SIMCE) están muy condicionados por factores socioeconómicos ${ }^{13}$ y que los niveles de movilidad social son bajos (Núñez y Risco, 2004). En este contexto, diversos autores 14 consideran que la educación es un determinante fundamental y atribuyen a variables socioeconómicas la actual situación de desigualdad en la calidad del aprendizaje. Dentro de estas variables se encuentra el NSE del que provienen los estudiantes, determinado tanto por su ingreso familiar como por el tipo de escuela a la que asistieron ${ }^{15}$.

\footnotetext{
${ }^{9}$ De acuerdo con información del Consejo de Rectores, en el proceso de admisión de 2012 se adscribieron al sistema único de admisión ocho universidades privadas, además de las 25 universidades tradicionales del CRUCH que participaban previamente.

10 Véase Contreras, Gallegos y Meneses (2009).

11 Véase DEMre (2012) y CRUCH (2012).

12 Véase Contreras, Gallegos y Meneses (2009).

13 Véase Mizala y Romaguera (2000).

14 Véanse Brunner y Elacqua (2003); Cornejo (2005).

15 Véase Contreras y Macías (2002).
} 
La presente investigación contribuye con nueva evidencia respecto de la repercusión de los determinantes socioeconómicos en el rendimiento académico. En primer lugar, se demuestra que los condicionantes socioeconómicos son estadísticamente significativos para explicar los niveles de logro tanto en la PSU como en el SIMCE. En segundo lugar, ocupando una base de datos de panel y siguiendo a un mismo individuo en dos momentos del tiempo (cuando rindió el SIMCE y la PSU), se vuelve a examinar el papel de los determinantes socioeconómicos, ahora controlando por dos categorías: alto y bajo NSE. La evidencia muestra que dos individuos de distinto NSE, medidos según ingreso familiar y escolaridad de los padres, y que además logran igual SIMCE en octavo básico, presentan una brecha de más de 70 puntos promedio cuatro años más tarde al rendir la PSU. Es decir, las brechas en logro académico no se reducen, sino que se amplifican, con el agravante de que los resultados en la PSU determinan el acceso a la

\section{II}

\section{Los datos}

En este artículo se utiliza una base de datos de panel, que incluye resultados de las pruebas SIMCE y PSU de la muestra completa de estudiantes que cursaron enseñanza media durante los años 2001 y 2004. La muestra total corresponde a 99.736 alumnos. Específicamente, se dispone del puntaje SIMCE obtenido en el año 2000 ( $8^{\circ}$ básico) y el puntaje PSU obtenido en el año 2004 (egresados de $4^{\circ}$ medio) por cada uno de los estudiantes. Es decir, se cuenta con los puntajes alcanzados por un mismo individuo en dos momentos diferentes del tiempo.

La variable NEM corresponde a las notas obtenidas durante la enseñanza media, y en este artículo se emplea para identificar la posición relativa del estudiante dentro de su establecimiento.

La base de datos incluye, además, una serie de variables correspondientes a características socioeconómicas de los estudiantes en el momento de rendir la PSU, y otras condiciones de los colegios a los que asistían cuando se tomó el SIMCE. De las variables socioeconómicas del individuo se consideran el género, el ingreso del grupo familiar y el nivel de educación de los padres. Con respecto al establecimiento, se tienen en cuenta su dependencia administrativa, su área geográfica y su modalidad educacional. educación superior y las altas tasas de retorno privado que esta supone ${ }^{16}$. Esto constituye evidencia de que el sistema educativo chileno no ha sido eficiente para compensar las diferencias de origen existentes entre los estudiantes.

Luego de esta Introducción, en la sección II se examinan los datos de panel que contienen los resultados de las pruebas SIMCE y PSU, a la luz de las variables correspondientes. En la sección III se utilizan los enfoques de función de producción y de valor agregado para analizar los resultados de dichas pruebas como función de un vector de variables socioeconómicas. En la sección IV se dan a conocer y se discuten los resultados obtenidos en la investigación y, por último, en la sección $\mathrm{V}$ se aportan las conclusiones.

\footnotetext{
${ }^{16}$ La evidencia aquí presentada también permite observar que dicha brecha es creciente en nivel de logro en el SIMCE.
}

Cabe señalar que de los estudiantes que rindieron el SIMCE se excluyó a quienes no rindieron la PSU el año 2004 y también a los que no lograron egresar de la enseñanza media en dicho año.

La descripción de las variables se presenta en el cuadro 1.

En el cuadro 2 se muestra la estadística descriptiva de las variables utilizadas.

Se observa que los puntajes del SIMCE van desde poco más de 100 puntos hasta poco menos de 400 , con un promedio cercano a 280 , mientras que los puntajes de la PSU van de los 150 a los 850 , con un promedio de 500. El puntaje bruto del sIMCE se ajusta o estandariza para obtener una media de 250 puntos y una desviación de 50 puntos. Esto se debe a que en la muestra del presente estudio solo se considera el puntaje SIMCE de aquellos estudiantes que rindieron la PSU en el año 2004. Es decir, en los datos no se incluye el puntaje SIMCE de quienes no lograron egresar, o no se presentaron a rendir la PSU en 2004.

La muestra está compuesta en un 54\% por mujeres y el promedio de la posición relativa en sus colegios de los estudiantes que rinden la PSU es de 0,52 con una desviación del $20 \%$. 
CUADRO 1

\section{Descripción de las variables}

\begin{tabular}{|c|c|}
\hline Variable & Descripción \\
\hline \multicolumn{2}{|l|}{ Puntaje SIMCE } \\
\hline Lenguaje & Puntaje obtenido en la prueba SIMCE de Lenguaje \\
\hline Matemáticas & Puntaje obtenido en la prueba SIMCE de Matemáticas \\
\hline Promedio & Promedio de los puntajes SIMCE de Lenguaje y Matemáticas \\
\hline \multicolumn{2}{|l|}{ Puntaje PSU } \\
\hline Lenguaje & Puntaje obtenido en la prueba PsU de Lenguaje \\
\hline Matemáticas & Puntaje obtenido en la prueba Psu de Matemáticas \\
\hline Promedio & Promedio de los puntajes Psu de Lenguaje y Matemáticas \\
\hline \multicolumn{2}{|l|}{ Variables del estudiante } \\
\hline Género & $1=$ Femenino, $0=$ Masculino \\
\hline NEM & Posición relativa en el colegio según notas de enseñanza media \\
\hline \multicolumn{2}{|l|}{ Variables socioeconómicas } \\
\hline Ingreso del grupo familiar & $\begin{array}{l}1=[\$ 0-\$ 278000] ; 2=[\$ 278000-\$ 834000] ; \\
3=[\$ 834000-\$ 1400000] ; 4=[\$ 1400000 \text { o más }]\end{array}$ \\
\hline $\begin{array}{l}\text { Nivel educacional } \\
\text { madre/padre }\end{array}$ & $\begin{array}{l}1=\sin \text { estudios, } 2=\text { básica incompleta, } 3=\text { básica completa, } \\
4=\text { media incompleta, } 5=\text { media completa, } 6=\text { CFT incompleta, } 7=\text { CFT completa, } 8=\text { universitaria incompleta, } \\
9=\text { universitaria completa, } 10=\text { otros estudios }\end{array}$ \\
\hline \multicolumn{2}{|l|}{ Variables del establecimiento } \\
\hline \multicolumn{2}{|l|}{ Dependencia: } \\
\hline - Municipal & $1=$ municipal, $0=$ otro \\
\hline - Particular subvencionado & $1=$ particular subvencionado, $0=$ otro \\
\hline - Particular pagado & $1=$ particular pagado, $0=$ otro \\
\hline Área geográfica & $1=$ rural, $0=$ urbana \\
\hline Modalidad & $0=$ técnico, $1=$ científico-humanista \\
\hline
\end{tabular}

Fuente: elaboración propia sobre la base de datos del Sistema de Medición de Calidad de la Educación (SIMCE) 2000 y la Prueba de Selección Universitaria (PSU) 2004.

CFT: centro de formación técnica.

NEM: promedio de notas de enseñanza media.

Se observa que la mayoría de los estudiantes pertenecen al primer tramo de ingresos (55\%), el $31 \%$ al segundo y solo un $7 \%$ a cada uno de los tramos superiores. Respecto de la dependencia del establecimiento, el $40 \%$ de los alumnos estudian en colegios municipales, el $43 \%$ en particulares subvencionados y solo un $17 \%$ en particulares pagados.

El nivel de educación promedio de los padres es de alrededor de 5, lo que corresponde a educación media completa, siendo levemente mayor en padres que en madres.

Finalmente, se aprecia que un $6 \%$ de alumnos pertenecen a áreas geográficas rurales, y el 77\% está en colegios con modalidad científico-humanista, mientras que el $22 \%$ restante pertenece a colegios con modalidad técnico-profesional.

Es importante considerar los puntajes promedio del SIMCE y la PSU para los distintos niveles de ingresos, así como el promedio de escolaridad de los padres y la composición de la muestra de los distintos tipos de colegios según su dependencia administrativa. La estadística descriptiva se presenta en el cuadro A1 del anexo.

Resalta claramente que a mayor tramo de ingresos, mayores son los puntajes promedio de los estudiantes en lenguaje y matemáticas tanto en el SIMCE como en la PSU. También se puede notar que los estudiantes de tramos de ingresos superiores tienen en promedio padres y madres con un mayor nivel de educación. Se constata, además, que la composición de alumnos en colegios municipales corresponde esencialmente al primer tramo de ingresos, mientras que en los subvencionados hay estudiantes sobre todo del $11^{\mathrm{er}} \mathrm{y}$ $2^{\circ}$ tramos; en los colegios particulares se revierte la situación, con alumnos principalmente del último y penúltimo tramos de ingresos. 
CUADRO 2

\section{Estadística descriptiva de las variables}

\begin{tabular}{|c|c|c|c|c|}
\hline Variables & Promedio & Desviación & Mínimo & Máximo \\
\hline \multicolumn{5}{|l|}{ Puntaje SIMCE } \\
\hline Lenguaje & 276 & 47 & 109 & 395 \\
\hline Matemáticas & 277 & 47 & 118 & 382 \\
\hline Promedio & 276 & 42 & 128 & 388 \\
\hline \multicolumn{5}{|l|}{ Puntaje PSU } \\
\hline Lenguaje PSU & 499 & 111 & 167 & 850 \\
\hline Matemáticas PSU & 501 & 112 & 150 & 850 \\
\hline Promedio & 500 & 104 & 198 & 840 \\
\hline \multicolumn{5}{|l|}{ Variables del estudiante } \\
\hline Mujer & $54 \%$ & 0,50 & 0 & 1 \\
\hline Calificación & 0,52 & 0,29 & 0,001 & 1 \\
\hline \multicolumn{5}{|l|}{ Variables socioculturales } \\
\hline \multicolumn{5}{|l|}{ Ingreso familiar: } \\
\hline- Tramo $1=[\$ 0-\$ 278000]$ & $55 \%$ & 0,50 & 0 & 1 \\
\hline- Tramo $2=[\$ 278000-\$ 834000]$ & $31 \%$ & 0,46 & 0 & 1 \\
\hline- Tramo $3=[\$ 834000-\$ 1400000]$ & $7 \%$ & 0,26 & 0 & 1 \\
\hline - Tramo 4 = [\$1 400000 o más $]$ & $7 \%$ & 0,26 & 0 & 1 \\
\hline Educación madre & 5,4 & 2,2 & 1 & 10 \\
\hline Educación padre & 5,6 & 2,4 & 1 & 10 \\
\hline \multicolumn{5}{|l|}{ Variables del colegio } \\
\hline \multicolumn{5}{|l|}{ Dependencia: } \\
\hline - Municipal & $40 \%$ & 0,49 & 0 & 1 \\
\hline - Particular subvencionado & $43 \%$ & 0,49 & 0 & 1 \\
\hline - Particular pagado & $17 \%$ & 0,37 & 0 & 1 \\
\hline Rural & $6 \%$ & 0,23 & 0 & 1 \\
\hline Modalidad científico-humanista & $77 \%$ & 0,42 & 0 & 1 \\
\hline \multicolumn{5}{|l|}{$N^{o}$ total de observaciones 99736} \\
\hline
\end{tabular}

Fuente: elaboración propia sobre la base de datos del Sistema de Medición de Calidad de la Educación (sIMCE) 2000 y la Prueba de Selección Universitaria (PSU) 2004.

\section{III}

\section{Metodología}

En este estudio se emplean los resultados obtenidos por un mismo individuo en dos momentos del tiempo en las pruebas del SIMCE y la PSU, que son instrumentos diseñados para evaluar el conocimiento de los estudiantes y que presentan entre sí una alta correlación $\left(75 \%{ }^{17}\right)$. En este trabajo se utiliza el enfoque de función de producción propuesto por McEwan y Carnoy (1999), a partir del cual se puede entender el resultado obtenido en las pruebas señaladas como el producto (output) de un conjunto de variables vinculadas al estudiante. Para ello se examinan los resultados de las pruebas SIMCE y PSU como función de un vector de variables socioeconómicas, que incluye

\footnotetext{
17 Véanse detalles en el cuadro A3 del anexo.
}

tipo de establecimiento, características del estudiante, y otras. Los resultados de esta regresión se presentan en la sección anexos (modelo general, cuadro A2 del anexo) y confirman el potencial explicativo de las variables socioeconómicas respecto de los puntajes obtenidos.

Comprobada la significancia estadística de los determinantes socioeconómicos en el desempeño de los estudiantes, el principal objeto de estudio es determinar hasta qué punto las condiciones exógenas a los individuos influyen en sus niveles de logro académico. Como ya se ha señalado, las pruebas PSU y SIMCE presentan una alta correlación, de manera que al controlar por una de ellas se supone que la repercusión del resto de las variables corresponde al efecto en el rendimiento a través del tiempo, dicho de otro modo: corresponde al efecto 
de segundo orden de las variables socioeconómicas en el nivel de logro. Para ello, se propone estimar una especificación motivada a partir de un modelo de efectos fijos, tomando los resultados de un mismo individuo en dos momentos del tiempo. Así, con el modelo propuesto se pretende eliminar, al menos en parte, el efecto de las variables no observables en los resultados.

Ahora bien, a fin de examinar a través del tiempo la contribución de las variables socioeconómicas se definieron dos variables dicotómicas: $N S E_{\text {bajo }}$ cuyo valor es 1 si el individuo tiene un ingreso familiar inferior a $\$ 278.000$, y $N S E_{\text {alto }}$ cuyo valor es 1 cuando el individuo tiene un ingreso familiar superior a $\$ 1.400 .000$. Además, se consideró de manera independiente una clasificación adicional de los estudiantes según el nivel educacional de los padres. Esta distinción se realizó desde una perspectiva cultural, siguiendo a Bourdieu y Passeron (1964): “... los alumnos que suelen tener éxito en la escuela pertenecen en gran mayoría a familias dotadas de un elevado nivel de instrucción, poseedoras por ende de un capital de tipo cultural que es heredado por sus hijos a través de la socialización que tiene lugar desde las edades más precoces en el espacio hogareño. Por lo cual, dichos niños cuentan con un bagaje de experiencias, saberes, formas de lenguaje y disposiciones que representan una ventaja considerable en el momento de ingresar a la escuela y abordar los aprendizajes que allí tienen lugar...". A partir de lo anterior, se crearon dos nuevas variables dicotómicas: nivel educacional bajo $\left(N E D_{b a j o}\right)$, cuyo valor es 1 para aquellos estudiantes cuyos padres no completaron la educación básica, y nivel educacional alto $\left(N E D_{\text {alto }}\right)$, que identifica a los estudiantes cuyos padres tienen estudios universitarios. El modelo principal es el siguiente:

$$
\begin{aligned}
& \text { PSU } U_{t}=\alpha+\beta_{1} \text { Part. Subvencionado }+ \\
& \beta_{2} \text { Part. Privado }+\beta_{2} \text { Modalidad }+ \\
& \beta_{4} \text { Género }+\beta_{5} \text { Educación Padres }
\end{aligned}
$$

En la ecuación (1) se tiene como variable dependiente el puntaje de la PSU (promedio de lenguaje y matemáticas), y como variables explicativas se consideraron: i) la dependencia del colegio (particular subvencionado, particular privado y municipal como variable omitida); ii) la modalidad de estudios (establecimientos científico-humanistas respecto de establecimientos técnicos); iii) género: que identifica el efecto de ser mujer; iv) la pertenencia a un sector rural; v) el nivel educacional promedio de los padres (desde $1=$ sin estudios a $10=$ estudios postuniversitarios); vi) el ingreso familiar agrupado en cuatro tramos, y por último, vii) la posición relativa del alumno en su establecimiento de acuerdo con su desempeño escolar (NEM).
En esta especificación también se incluye como regresor el puntaje obtenido por el mismo estudiante, cuatro años antes, en la prueba SIMCE. De esta manera se pretende analizar el efecto adicional de las variables que ya están capturadas implícitamente en el puntaje SIMCE, considerando que:

\section{SIMCE $_{t-4}=\alpha^{-}+\beta_{1}^{-}$Part. Subvencionado+ $\beta_{2}^{-}$Part.Privado $+\beta_{2}^{-}$Modalidad $+\beta_{2}^{-}$Género + \\ $\beta_{5}^{-}$Educación Padres}

También en la ecuación (1) se refleja un enfoque de valor agregado en la estimación de una función de producción de logros. Al respecto, en la literatura se señala que la evidencia basada en este tipo de especificaciones es generalmente preferida en comparación con aquella obtenida a partir de información contemporánea (Hanushek, 1996; Krueger, 2000; Todd y Wolpin, 2003), esto en tanto permite limpiar, al menos parcialmente, el efecto de variables no observables en los resultados.

De acuerdo con Todd y Wolpin (2003), una especificación de valor agregado requiere de supuestos sobre el comportamiento de variables no observables, y por lo tanto está expuesta a problemas de endogeneidad. Una de las principales críticas a este tipo de modelos es que si el comportamiento de los agentes es óptimo, entonces se debiese esperar que las familias tomen decisiones a partir del punto de referencia (baseline achievement) obtenido por los alumnos ( $\operatorname{SINCE}_{\mathrm{t}-4}$ en este caso). En Chile, sin embargo, los puntajes del SIMCE no son informados a las familias, por lo que los padres no pueden tomar decisiones a partir de este resultado. Lo anterior da mayor sentido a la especificación aportada en este estudio, pues aminora el sesgo por endogeneidad.

En conclusión, con este modelo se pretende evaluar el efecto y la significancia estadística de los condicionantes socioeconómicos respecto del nivel de logro académico. Además, esta especificación permite evaluar si la repercusión de estos factores es creciente a través del tiempo.

Ahora bien, junto con la ecuación (1) también se han definido los siguientes modelos

$$
\begin{gathered}
P S U_{t}=\alpha+\beta_{1} \operatorname{SIMCE}_{t-4}+ \\
\beta_{2} N S E_{\text {alto }}+\beta_{3} N S E_{\text {bajo }}+\varepsilon_{t} \\
P S U=\alpha+\beta_{1} S_{I M C E_{t-4}+\beta_{2} N S E_{\text {alto }}+}+ \\
\beta_{3} N S E_{\text {bajo }}+\beta_{4} N S E_{\text {alto }} S_{M C E_{t-4}}+ \\
\beta_{5} N S E_{\text {bajo }} \operatorname{SIMCE}_{t-4}+\varepsilon_{t}
\end{gathered}
$$


La ecuación (2) permite testear la existencia de un diferencial en el rendimiento en la PSU conducido exclusivamente por el nivel socioeconómico (NSE). Para ello se controla por el rendimiento en el SIMCE. Mientras que en la ecuación (3) se incluyen variables interactivas entre el nivel de logro en el SIMCE y el NSE; con ello se pretende relajar el supuesto de que la diferencia entre el rendimiento de ambos grupos, si es que existe, es constante. Se han considerado estas especificaciones con el objeto de mostrar de manera clara el efecto del NSE alto y bajo en los resultados de la PSU, controlando por el SIMCE ${ }^{18}$.

\footnotetext{
${ }^{18}$ En las ecuaciones (2) y (3) no se ha considerado el vector de regresores de la ecuación (1), puesto que la mayoría de estos controles están altamente correlacionados con el promedio de NSE. Ahora bien, el signo y significancia de los resultados se mantienen si se incluyen todos los controles; esta estimación se reporta en el cuadro A4 del anexo.
}

\section{IV}

\section{Resultados y discusión}

Todas las variables resultaron ser significativas y los efectos van en la misma dirección que en el modelo clásico desarrollado por Mizala y Romaguera (2000), con lo que se reafirma que en Chile los condicionantes socioeconómicos repercuten en el nivel de logro académico, pero además se presenta nueva evidencia de que estos factores influyen en el rendimiento de manera creciente a través del tiempo. Lo anterior podría atribuirse a que los individuos con mejores condiciones económicas pueden seguir realizando a través del tiempo una mayor inversión y, por tanto, resulta esperable un mayor retorno (entendido como un puntaje más elevado). No obstante, lo central aquí es que una exposición más alta a un contexto socioeconómico desfavorable supone un aumento creciente de las restricciones enfrentadas para obtener un buen puntaje en la PSU y acceder con ello a la educación superior.

Las estimaciones del modelo especificado en la ecuación 1 se muestran en el cuadro 3.

CUADRO 3

Determinantes de la Prueba de Selección Universitaria (PSU)

\begin{tabular}{|c|c|c|c|}
\hline Variables & PSU Mat & PSU Leng & PSU \\
\hline Subvencionados & $7,542 * * *$ & $10,93 * * *$ & $8,505^{* * *}$ \\
\hline Privados & $33,35 * * *$ & $32,91 * * *$ & $30,46 * * *$ \\
\hline Modalidad & $42,36 * * *$ & $35,87 * * *$ & $37,62 * * *$ \\
\hline Mujer = 1 & $-33,54 * * *$ & $-21,35^{* * *}$ & $-27,45^{* * *}$ \\
\hline Educación padres & $5,068 * * *$ & $6,488 * * *$ & $5,117 * * *$ \\
\hline Rural & $-19,66 * * *$ & $-21,03 * * *$ & $-18,98 * * *$ \\
\hline Ingreso familiar & $10,46^{* * *}$ & $8,943 * * *$ & $9,095^{* * *}$ \\
\hline NEM (relativo) & $95,87 * * *$ & $94,38 * * *$ & $84,80 * * *$ \\
\hline SIMCE Mat & $1,174 * * *$ & & \\
\hline SIMCE Leng & & $1,157 * * *$ & \\
\hline SIMCE & & & $1,345^{* * *}$ \\
\hline Constante & $58,51 * * *$ & $54,76 * * *$ & $18,81 * * *$ \\
\hline $\mathrm{N}^{\circ}$ observaciones & 87417 & 87417 & 87417 \\
\hline $\mathrm{R}^{2}$ & 0,647 & 0,601 & 0,713 \\
\hline
\end{tabular}

Fuente: elaboración propia sobre la base de datos del Sistema de Medición de Calidad de la Educación (sIMCE) 2000 y la Prueba de Selección Universitaria (PSU) 2004.

Nota:***p $<0,01$.

PSU Mat: PSU Matemáticas.

PSU Leng: PSU Lenguaje.

SIMCE Mat: SIMCE Matemáticas.

SIMCE Leng: SIMCE Lenguaje.

NEM: Promedio de notas de enseñanza media. 
En virtud de los resultados obtenidos se consideró pertinente estudiar con más detalle la repercusión de las variables socioeconómicas, para ello se estimó el modelo especificado en la ecuación 2. Los resultados observados son los siguientes:

CUADRO 4

\section{Prueba de Selección Universitaria (PSU) según ingreso}

\begin{tabular}{lc}
\hline \multicolumn{1}{c}{ Variables } & PSU \\
\hline SIMCE & $1,710 * * *$ \\
NSE alto & $43,08 * * *$ \\
NSE bajo & $-31,30 * * *$ \\
Constante & $41,50 * * *$ \\
$\mathrm{~N}^{\circ}$ observaciones & 99706 \\
$\mathrm{R}^{2}$ & 0,617 \\
\hline
\end{tabular}

Fuente: elaboración propia sobre la base de datos del Sistema de Medición de Calidad de la Educación (SIMCE) 2000 y la Prueba de Selección Universitaria (PSU) 2004.

Nota: $* * * \mathrm{p}<0,01$.

NSE: nivel socioeconómico.

Se observa directamente que en promedio, para el mismo desempeño en el SIMCE, los individuos de ${ }_{N S E_{\text {alto }}}$ presentan mejor rendimiento en la PSU que los individuos de $N S E_{\text {bajo }}$, con una diferencia promedio de 70 puntos.

Una limitación de este modelo ha sido asumir que la diferencia entre el rendimiento de ambos grupos es constante; por ello se incluye una variable interactiva entre el nivel de logro en el SIMCE y el condicionante socioeconómico, que tiene por objeto capturar el retorno adicional de cada punto obtenido en el SIMCE de acuerdo con el NSE de los estudiantes. Este retorno es el que proviene de la variación marginal con respecto al desempeño en dicha prueba. Entonces, de estimarse el modelo especificado en la ecuación 3, los resultados obtenidos son:

CUADRO 5

Prueba de Selección Universitaria (PSU) según ingreso e interactiva con el SIMCE

\begin{tabular}{lc}
\hline Variables & PSU \\
\hline SIMCE & $1,784 * * *$ \\
NSE alto & $65,37 * * *$ \\
NSE bajo & 3,637 \\
NSE alto SIMCE & $-0,0782 * * *$ \\
NSE bajo SIMCE & $-0,126 * * *$ \\
Constante & 20,63 \\
$\mathrm{~N}^{\circ}$ observaciones & 99706 \\
$\mathrm{R}^{2}$ & 0,617 \\
\hline
\end{tabular}

Fuente: elaboración propia sobre la base de datos del Sistema de Medición de Calidad de la Educación (SIMCE) 2000 y la Prueba de Selección Universitaria (PSU) 2004.

Nota: $* * * \mathrm{p}<0,01$.

NSE: nivel socioeconómico.

Ahora bien, dados los parámetros estimados en las regresiones anteriores, y para analizar de mejor manera los resultados, se muestran aquí los puntajes en la PSU esperados según nivel socioeconómico:

$$
\begin{aligned}
& P S U\left(N S E_{\text {alto }}=1\right)=85,9+1,71 * \text { SIMCE } \\
& P S U\left(N S E_{\text {bajo }}=1\right)=24,3+1,66 * \text { SIMCE }
\end{aligned}
$$

Cabe mencionar que se testeó la significancia estadística de ambas pendientes ${ }^{19}$, y se descartó con

${ }^{19}$ La prueba de significancia se presenta en el anexo.

GRÁFICO 1

PSU en comparación con el SIMCE según nivel socioeconómico (NSE)

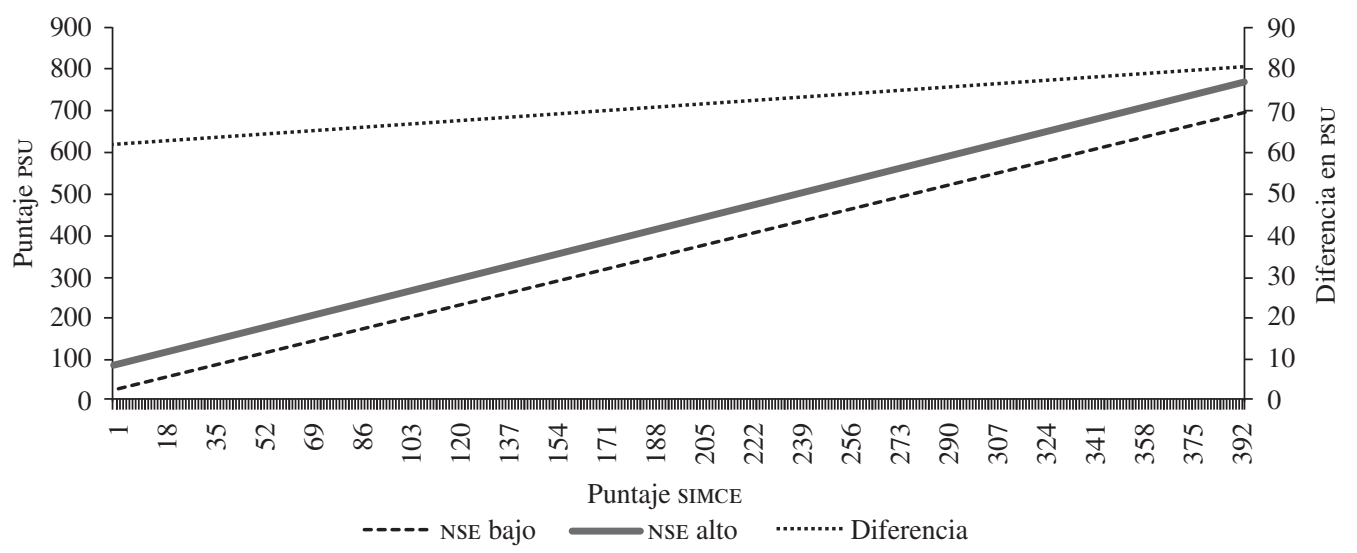

Fuente: elaboración propia a partir de resultados del cuadro 5, sobre la base de datos del Sistema de Medición de Calidad de la Educación (SIMCE) 2000 y la Prueba de Selección Universitaria (PSU) 2004. 
un $99 \%$ de confianza la posibilidad de que no existiese diferencia significativa entre ellas.

En promedio, para un mismo resultado en el SIMCE, los individuos de $N E_{b a j o}$ tienen un peor rendimiento en la PSU con respecto a todos los niveles de puntaje en el SIMCE. Además, mientras mayor es el desempeño en el SIMCE mayor es el efecto del NSE en el puntaje de la PSU.

La brecha de acuerdo con el NSE es cercana a los 70 puntos de la PSU, y va en aumento desde 60 puntos en los alumnos con peor rendimiento en el SIMCE hasta más de 80 puntos en aquellos con mejores resultados.

Entonces, si se toman de manera aleatoria dos individuos con igual desempeño académico en un momento del tiempo (SIMCE), uno de NSE alto y el otro de $N S E_{\text {bajo, }}$, se observa que el primero obtiene 70 puntos más que el segundo en sus resultados de la PSU. Es más, esta brecha en puntajes va aumentando en estudiantes con mejores resultados en el SIMCE, es decir, a mayor logro académico mayor es también el castigo o premio por nivel de ingresos.

Con ello se ha mostrado que existe, para todos los estudiantes con igual puntaje en el SIMCE, una brecha según NSE respecto del rendimiento en la PSU, siendo mayor el nivel de logro en dicha prueba para los individuos con condicionantes socioeconómicas favorables en comparación con aquellos en situación vulnerable, y siendo creciente esta brecha al aumentar el resultado del SIMCE.

También se ha estimado el modelo especificado en la ecuación (3) considerando el NSE en términos culturales, esto es, vinculándolo a la escolaridad de los padres. Los resultados son los siguientes:

CUADRO 6

PSU según nivel educacional de los padres e interactiva con el SIMCE

\begin{tabular}{lc}
\hline Variables & PSU \\
\hline SIMCE & $1,742 * * *$ \\
Ned bajo & $16,57 * *$ \\
Ned alto & $37,67 * * *$ \\
Ned bajo SIMCE & $-0,175^{* * *}$ \\
Ned alto SIMCE & $0,0293^{*}$ \\
Constante & $13,88 * * *$ \\
$N^{\circ}$ observaciones & 87638 \\
$\mathrm{R}^{2}$ & 0,605 \\
\hline
\end{tabular}

Fuente: elaboración propia sobre la base de datos del Sistema de Medición de Calidad de la Educación (SIMCE) 2000 y la Prueba de Selección Universitaria (PSU) 2004.

Nota:***p $<0,01, * * \mathrm{p}<0,05, * \mathrm{p}<0,1$

Ned: nivel educacional.

Se puede notar que al igual que con el nivel de ingresos, los estudiantes con padres poco educados en comparación con los con padres muy educados presentan una importante brecha en puntajes de la PSU, para igual resultado en la prueba SIMCE.

Esta brecha es también creciente en el desempeño respecto de la prueba SIMCE, es decir, a mayor puntaje en esta medición más relevante es la diferencia entre ambos

GRÁFICO 2

\section{Puntaje en la PSU comparado con puntaje en el SIMCE según educación de los padres}

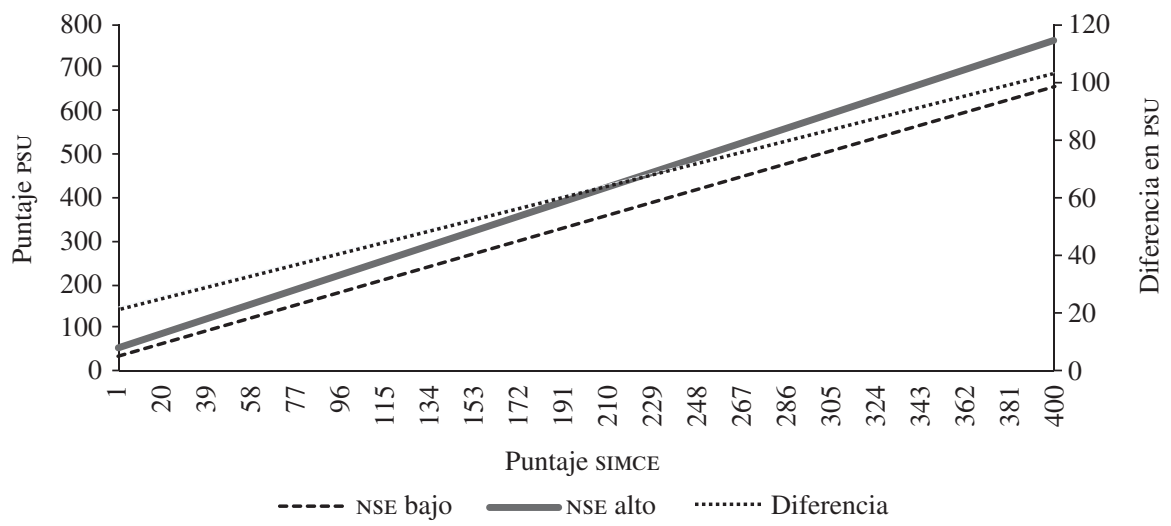

Fuente: elaboración propia a partir de resultados del cuadro 5, sobre la base de datos del Sistema de Medición de Calidad de la Educación (SIMCE) 2000 y la Prueba de Selección Universitaria (PSU) 2004.

NSE: nivel socioeconómico. 
grupos, desde 20 puntos para aquellos que obtuvieron un puntaje SIMCE más bajo, hasta 100 puntos para aquellos con mayor logro en el SIMCE. En promedio se tiene una diferencia de 60 puntos entre los puntajes de individuos de $\operatorname{Ned}_{\text {bajo }}$ y Ned alto $_{\text {. }}$

En resumen, los resultados muestran que los factores socioeconómicos repercuten de manera significativa en el puntaje obtenido tanto en la prueba SIMCE como en la PSU. Además, el efecto de estos factores es significativamente creciente a través del tiempo, esto es: para un mismo individuo se generan diferencias en el nivel de logro en la PSU respecto del nivel de logro en el SIMCE. Así, al controlar por NSE, la evidencia muestra brechas promedio de 70 y 60 puntos en la PSU, para un mismo nivel de logro en el SIMCE (considerando el NSE según ingresos y según educación de los padres, respectivamente).

Cabe considerar que el efecto estimado del NSE en los resultados está sujeto a sesgo de selección, pues en los datos utilizados solo se consideran aquellos estudiantes que rindieron la PSU en 2004. Luego, si se toma en cuenta que quienes llegan a la PSU son generalmente estudiantes de mayor NSE, los efectos que aquí hemos presentado estarían potencialmente subestimados.

Ahora bien, la significancia de los resultados reportados en el presente trabajo es robusta a una especificación por mínimos cuadrados en dos etapas, con un estimador de máxima verosimilitud con información completa (Full-Information Maximum Likelihood) ${ }^{20}$. En efecto, para comprobar la robustez de las estimaciones efectuadas en este estudio se empleó una nueva cohorte de alumnos que rindieron la prueba SIMCE (2006) y la PSU (2008), obteniéndose resultados consistentes (en la dirección del efecto y su significancia) con los reportados en el presente trabajo ${ }^{21}$.

20 De acuerdo con Puhani (2000), este estimador es preferible al tradicional método de Heckman en dos etapas.

${ }^{21}$ Los resultados de la ecuación (3) con la nueva base de datos pueden verse en el cuadro A5 del anexo.
Para efectos de estimación se consideró como variable de identificación las expectativas de estudios futuros que tienen los padres respecto del estudiante en el momento de rendir el SIMCE. La intuición es que las expectativas de los padres influyen en la decisión de rendir o no la PSU, pero no afectan el resultado que obtienen los alumnos en dicha prueba, dado que aquellos padres que no esperan que su hijo alcance estudios universitarios, no proporcionarán los medios o incentivos necesarios para que el alumno se inscriba en la PSU, todo ello al margen de las capacidades del estudiante. Más aún, las expectativas de los padres podrían estar revelando decisiones de planificación familiar tales como que el estudiante ingrese al mercado laboral una vez terminados sus estudios obligatorios a fin de ayudar económicamente a su familia. Si bien pudiese existir debate acerca de la elección de la variable de identificación, puede considerarse que es la mejor dentro de las disponibles.

La estimación se reporta en el cuadro A6 del anexo, y confirma que - de modo condicional a este sesgo de selección - los resultados se mantienen, es decir, que existe una brecha significativa en el nivel de logro en la PSU según NSE, controlando por el resultado en el SIMCE.

Los resultados aquí presentados se traducen en una potencial pérdida de talentos, ya que una diferencia de 60 puntos en la PSU genera restricciones de acceso a la educación superior. Además, en Chile el puntaje obtenido no solo determina las posibilidades de acceso a distintas carreras dentro de cada plantel, sino que también restringe el acceso a fuentes de financiamiento, principalmente subsidios estatales.

Se aprecia que la formación de capital humano se da preferentemente en aquellos que ya cuentan con mayores recursos, existiendo cierta complementariedad entre el capital pecuniario (y cultural) y el capital humano, agudizándose con ello la desigualdad en la distribución de los ingresos a través del tiempo.

muestran que utilizando dos criterios de NSE (pecuniario y cultural) existe una brecha significativa y creciente en los resultados de la PSU. En efecto, dos alumnos de

octavo básico con igual resultado en la prueba SIMCE,
Mediante una base de datos de panel se ha evidenciado que existe una influencia positiva, y creciente en el tiempo, de los factores socioeconómicos en el desempeño de los estudiantes. Junto con ello, los resultados 
provenientes de distinto NSE, muestran brechas del orden de 70 puntos promedio en la PSU. Además, esta brecha se amplía a medida que aumenta el puntaje SIMCE inicial.

Suponiendo que las variables no observables (habilidad, motivación, y otros) se mantienen constantes a través del tiempo, se puede señalar que el nivel de logro en la PSU, y por ende la posibilidad de acceso a la educación superior, están condicionados por factores exógenos al estudiante, lo que restringe la expresión de talentos en el ámbito académico y productivo.

En este trabajo se subraya la necesidad de evaluar el carácter potencialmente regresivo de los instrumentos diseñados para restringir el acceso a la educación terciaria. El asunto es relevante para Chile y América Latina en virtud de dos características estructurales: los altos retornos que supone la educación superior y los elevados niveles de desigualdad en la distribución del ingreso.

En Chile, el actual exceso de demanda por trabajadores con mayor capital humano abre espacio al desarrollo de políticas en favor de la igualdad, las que debiesen garantizar restricciones mínimas y homogéneas al acceso a la educación superior, asignando capital humano a quienes son intelectualmente más productivos y no a quienes por azar enfrentan menos restricciones económicas o culturales.

Se espera que la evidencia presentada motive futuras investigaciones con respecto a los mecanismos de selección y promueva la innovación en materia de políticas que promuevan la movilidad social, puesto que con ello es posible alcanzar más crecimiento con mayor igualdad.

ANEXOS

Estadística descriptiva

CUADRO A1

Características según el nivel socioeconómico (NSE)

\begin{tabular}{|c|c|c|c|c|c|c|}
\hline Ingreso familiar & & Tramo 1 & Tramo 2 & Tramo 3 & Tramo 4 & Total \\
\hline \multirow[t]{2}{*}{ SIMCE } & Matemáticas & 266,8 & 281,6 & 297,7 & 310,0 & 276,8 \\
\hline & Lenguaje & 267,3 & 280,4 & 293,7 & 303,2 & 275,9 \\
\hline \multirow[t]{2}{*}{ PSU } & Matemáticas & 466,9 & 518,0 & 572,3 & 615,2 & 501,1 \\
\hline & Lenguaje & 466,6 & 515,5 & 564,4 & 602,4 & 498,7 \\
\hline \multirow[t]{2}{*}{ Educación } & Padre & 4,45 & 6,33 & 7,91 & 8,49 & 5,62 \\
\hline & Madre & 4,37 & 6,09 & 7,32 & 7,94 & 5,38 \\
\hline \multirow[t]{4}{*}{ Dependencia } & Municipal & 29803 & 9289 & 931 & 255 & 40278 \\
\hline & Subvencionado & 23335 & 16077 & 2308 & 720 & 42440 \\
\hline & Particular & 1352 & 5243 & 4171 & 6252 & 17018 \\
\hline & Total & 54490 & 30609 & 7410 & 7227 & 99736 \\
\hline
\end{tabular}

Fuente: elaboración propia sobre la base de datos del Sistema de Medición de Calidad de la Educación (SIMCE) 2000 y la Prueba de Selección Universitaria (PSU) 2004.

\section{- Modelo general}

Se presentan los resultados del modelo general de función de producción del puntaje, tanto del sIMCE como de la Psu, sobre la base de las variables socioeconómicas ya explicadas y utilizando los datos del SIMCE 2000 y de la PSU 2004.

El modelo es el siguiente:

$$
\text { Puntaje }=a+\beta_{1} \text { Part.Subvencionado }+\beta_{2} \text { Part.Privado }+\beta_{2} \text { Modalidad }+\beta_{4} \text { Género }+\beta_{5} \text { EducaciónPadres }+\beta_{6} 1
$$

Los parámetros de este modelo fueron estimados tanto para las pruebas de lenguaje y matemáticas, como para el promedio de estas, considerando separadamente los resultados del SIMCE y la PSU. Los resultados son los siguientes: 
Determinantes del rendimiento en las pruebas SIMCE y PSU

\begin{tabular}{|c|c|c|c|c|c|c|}
\hline Variables & PSU Mat & PSU Leng & PSU & SIMCE Mat & SIMCE Leng & SIMCE \\
\hline Subvencionados & $12,03 * * *$ & $15,90 * * *$ & $13,97 * * *$ & $3,828 * * *$ & $4,292 * * *$ & $4,060 * * *$ \\
\hline Privados & $52,43 * * *$ & $48,59 * * *$ & $50,51 * * *$ & $16,26 * * *$ & $13,56 * * *$ & $14,91 * * *$ \\
\hline Modalidad & $51,37 * * *$ & $46,10 * * *$ & $48,74 * * *$ & $7,670 * * *$ & $8,851 * * *$ & 8,261 *** \\
\hline Mujer $=1$ & $-42,49 * * *$ & $-13,28 * * *$ & $-27,89 * * *$ & $-7,637 * * *$ & $6,962 * * *$ & $-0,337$ \\
\hline Educación padres & $9,161 * * *$ & $10,93 * * *$ & $10,05 * * *$ & $3,482 * * *$ & $3,845^{* * * *}$ & 3,664 *** \\
\hline Rural & $-28,52 * * *$ & $-29,80 * * *$ & $-29,16^{* * * *}$ & $-7,573 * * *$ & $-7,597 * * *$ & $-7,585^{* * * *}$ \\
\hline Ingreso familiar & $15,39 * * *$ & $11,93 * * *$ & $13,66^{* * *}$ & $4,216 * * *$ & $2,580 * * *$ & $3,398 * * *$ \\
\hline NEM (relativo) & $165,9 * * *$ & $158,5 * * *$ & $162,2 * * *$ & $59,62 * * *$ & $55,40 * * *$ & $57,51 * * *$ \\
\hline Constante & $309,5 * * *$ & $294,5 * * *$ & $302,0 * * *$ & $213,7 * * *$ & $207,2 * * *$ & $210,5 * * *$ \\
\hline $\mathrm{N}^{\circ}$ observaciones & 87441 & 87441 & 87441 & 87417 & 87417 & 87417 \\
\hline $\mathrm{R}^{2}$ & 0,465 & 0,412 & 0,493 & 0,255 & 0,224 & 0,282 \\
\hline
\end{tabular}

Fuente: elaboración propia sobre la base de datos del Sistema de Medición de Calidad de la Educación (sIMcE) 2000 y la Prueba de Selección Universitaria (PSU) 2004.

Nota: ***p $<0,01$.

SIMCE Leng: SIMCE Lenguaje.

SIMCE Mat: SIMCE Matemáticas.

Casi todas las variables resultaron ser significativas a un 99\% de confianza y el nivel de ajuste de los modelos también resultó ser muy bueno, en consideración de que se omiten una serie de variables de difícil medición, como lo es la habilidad.

Estos resultados concuerdan con la teoría respecto de la influencia de los factores socioculturales en el rendimiento escolar. En general, se constata que la educación de los padres, el ingreso familiar y el promedio NEM influyen positivamente en el nivel de logro en las pruebas. Asimismo, colegios de dependencia particular pagada, particular subvencionada y de modalidad científico-humanista influyen positivamente en el nivel de logro en todas las pruebas. A su vez, la condición geográfica de ruralidad afecta negativamente el desempeño en las pruebas, lo que podría obedecer a la menor oferta de escuelas en el sector rural, así como a las dificultades para trasladarse al lugar de estudio. Finalmente, la evidencia muestra que ser mujer repercute negativamente en el nivel de logro alcanzado, con excepción de la prueba simce de lenguaje.

\section{- Correlación SIMCE-PSU según cuadro A3:}

CUADRO A3

\section{Correlación entre las pruebas PSU y SIMCE}

\begin{tabular}{lcc}
\hline Correlación & PSU & SIMCE \\
\hline PSU & 1 & \\
SIMCE & 0,7592 & 1 \\
\hline
\end{tabular}

Fuente: elaboración propia sobre la base de datos del Sistema de Medición de Calidad de la Educación (SIMCE) 2000 y la Prueba de Selección Universitaria (PSU) 2004.

\section{- Prueba de significancia y repercusión del SIMCE según NSE}

La hipótesis nula, $H_{o}$, está dada por la siguiente expresión

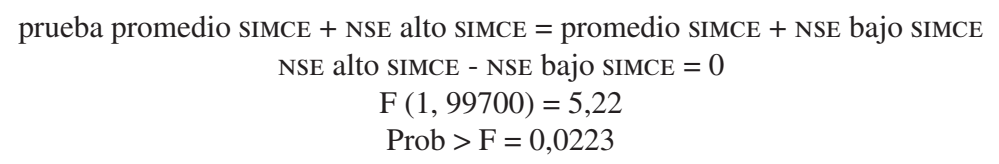

Se rechaza la hipótesis nula de no significancia con un 97\% de confianza. 
— Gráfico A1 de puntajes SIMCE y PSU de la población completa, considerando nSE según ingreso familiar: GRÁFICO A1 PSU en comparación con el SIMCE según NSE

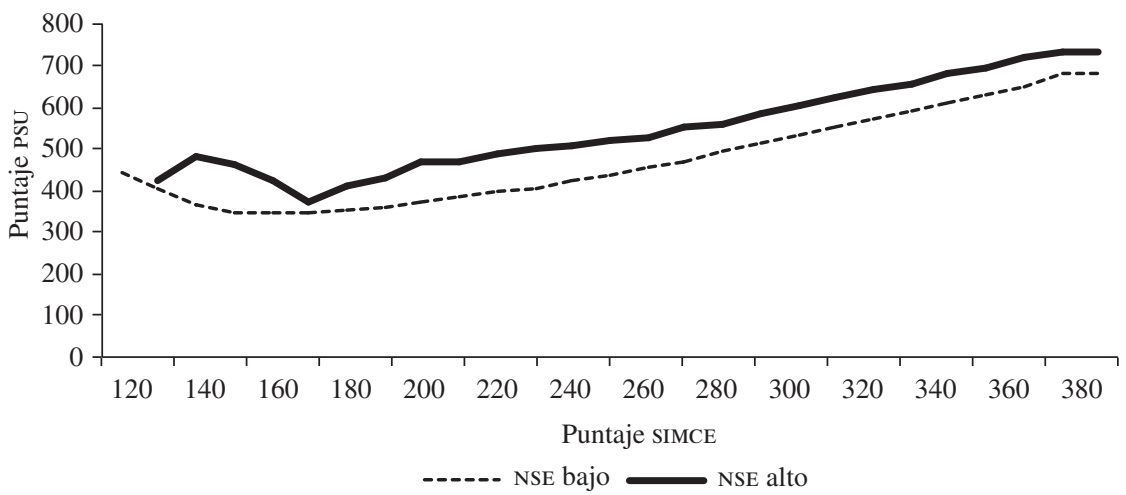

Fuente: elaboración propia sobre la base de datos del Sistema de Medición de Calidad de la Educación (SIMCE) 2000 y la Prueba de Selección Universitaria (PSU) 2004.

NSE: nivel socioeconómico.

— Gráfico A2 de puntajes SIMCE y PSU de la población completa considerando nSE, según la escolaridad de los padres: GRÁFICO A2 PSU en comparación con el SIMCE, según educación de los padres

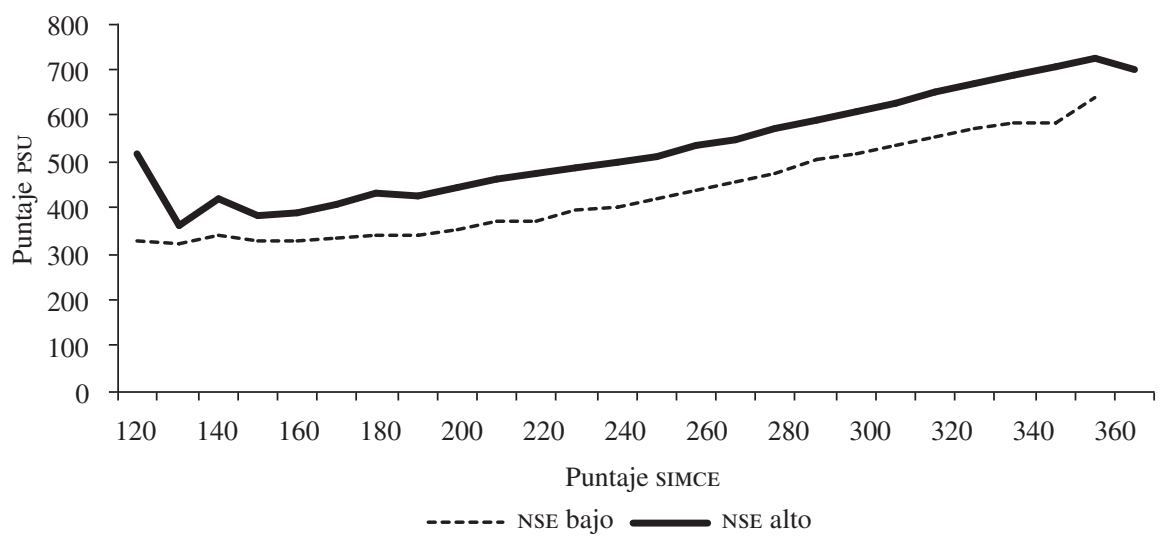

Fuente: elaboración propia sobre la base de datos del Sistema de Medición de Calidad de la Educación (SIMCE) 2000 y la Prueba de Selección Universitaria (PSU) 2004.

NSE: nivel socioeconómico.

— Ecuación (3) incluidos controles de la ecuación (1).

CUADRO A4

PSU según NSE, interactiva con el SIMCE y controles

\begin{tabular}{lccc}
\hline Variables & PSU Mat & PSU Leng & PSU \\
\hline NSE alto & $-39,52 * * *$ & $-71,29 * * *$ & $26,28 * * *$ \\
NSE bajo & $-47,78 * * *$ & $-106,1 * * *$ & 4,022 \\
NSE alto SIMCE & $0,204 * * *$ & $0,302 * * *$ & $-0,0247$
\end{tabular}


(Conclusión)

\begin{tabular}{|c|c|c|c|}
\hline Variables & PSU Mat & PSU Leng & PSU \\
\hline NSE bajo SIMCE & $0,138 * * *$ & $0,356 * * *$ & $-0,0440 * * *$ \\
\hline SIMCE & & & $1,371 * * *$ \\
\hline SIMCE Mat & $1,106 * * *$ & & \\
\hline SIMCE Leng & & $0,993 * * *$ & \\
\hline Subvencionados & $7,501 * * *$ & $10,51 * * *$ & $8,727 * * *$ \\
\hline Privados & $35,39 * * *$ & $35,24 * * *$ & $31,39 * * *$ \\
\hline Modalidad & $42,48 * * *$ & $35,85 * * *$ & $37,83 * * *$ \\
\hline Mujer = 1 & $-34,04 * * *$ & $-19,90 * * *$ & $-27,59 * * *$ \\
\hline Educación padres & $5,342 * * *$ & $6,733 * * *$ & $5,367 * * *$ \\
\hline Rural & $-19,12 * * *$ & $-19,75 * * *$ & $-19,02 * * *$ \\
\hline NEM (relativo) & $95,16^{* * *}$ & $92,12 * * *$ & $84,70 * * *$ \\
\hline Constante & $97,97 * * *$ & $118,2 * * *$ & $28,41 * * *$ \\
\hline $\mathrm{N}^{\circ}$ observaciones & 87417 & 87417 & 87417 \\
\hline $\mathrm{R}^{2}$ & 0,647 & 0,606 & 0,713 \\
\hline
\end{tabular}

Fuente: elaboración propia sobre la base de datos del Sistema de Medición de Calidad de la Educación (SIMCE) 2000 y la Prueba de Selección Universitaria (PSU) 2004.

Nota: $* * * \mathrm{p}<0,01$.

NSE: nivel socioeconómico.

NEM: promedio de notas de la enseñanza media.

SIMCE Leng: SIMCE Lenguaje.

SIMCE Mat: SIMCE Matemáticas.

\section{— Modelo mínimos cuadrados en 2 etapas (MC2E) para resolver sesgo de selección.}

Empleando una cohorte de alumnos que han rendido el SIMCE en el año 2006 y la PSU en 2008, se obtienen resultados consistentes con los reportados en el presente trabajo. En el cuadro A5 se reportan los resultados de estimar la ecuación (3) con esta nueva base de datos.

CUADRO A5

PSU según ingresos e interactiva con el SIMCE

\begin{tabular}{lccc}
\hline Variables & PSU Leng & PSU Mat & PSU \\
\hline SIMCE & & & $1,578^{* * *}$ \\
NSE bajo & $-61,01 * * *$ & $22,76^{* * *}$ & $33,16^{* * *}$ \\
NSE alto & $14,29 * * *$ & $24,00^{* * *}$ & $69,23^{* * *}$ \\
NSE bajo SIMCE & $0,0971^{* * *}$ & $-0,217 * * *$ & $-0,252^{* * *}$ \\
NSE alto SIMCE & $0,167 * * *$ & $0,126^{* * *}$ & $-0,0409^{* * *}$ \\
SIMCE Leng & $1,512^{* * *}$ & $1,286^{* * * *}$ & $143,0^{* * *}$ \\
SIMCE Mat & $84,01 * * *$ & 144762 & $64,87^{* * *}$ \\
Constante & 145413 & 0,656 & 144624 \\
$\mathrm{~N}^{\circ}$ observaciones & 0,632 & 0,738 \\
$\mathrm{R}^{2}$ & &
\end{tabular}

Fuente: elaboración propia sobre la base de datos del Sistema de Medición de Calidad de la Educación (SIMCE) 2006 y la Prueba de Selección Universitaria (PSU) 2008.

Nota: $* * * \mathrm{p}<0,01$.

SIMCE Leng: SIMCE Lenguaje.

SIMCE Mat: SIMCE Matemáticas.

NSE: nivel socioeconómico.

Resalta que el sentido y la significancia de los principales resultados se mantienen empleando esta nueva base de datos. Además, con esta cohorte es posible resolver el problema de sesgo de selección a partir de una estimación por máxima verosimilitud con información completa, símil de la metodología de MC2E propuesta en Heckman (1979). 
Para efectos de estimación se han considerado como variable de identificación las expectativas de estudios futuros que tienen los padres respecto del estudiante en el momento de rendir el simcE. Los resultados se reportan en el cuadro A6.

CUADRO A6

PSU según NSE e interactiva con el SIMCE corregido por modelo de selección de Heckman

\begin{tabular}{|c|c|c|c|c|c|c|}
\hline Variables & PSU Leng & Selección & PSU Mat & Selección & PSU & Selección \\
\hline Expectativas & & $0,131 * * *$ & & $0,123 * * *$ & & $0,118 * * *$ \\
\hline SIMCE & & & & & $1,524 * * *$ & $0,00990 * * *$ \\
\hline NSE bajo & $-38,55^{* * * *}$ & $-0,592 * * *$ & $30,22 * * *$ & $-0,353 * * *$ & $38,91 * * *$ & $0,0739 *$ \\
\hline NSE alto & $-15,19 * * *$ & $0,380 * * *$ & $9,247 * *$ & $0,372 * * *$ & $50,88 * * *$ & $0,898 * * *$ \\
\hline NSE bajo SIMCE & $0,0372 * * *$ & $0,00120 * * *$ & $-0,236 * * *$ & 0,000214 & $-0,264 * * *$ & $-0,00153$ *** \\
\hline NSE alto SIMCE & $0,241 * * *$ & $0,000878 * *$ & $0,168 * * *$ & 0,000434 & 0,0126 & $-0,00150 * * *$ \\
\hline SIMCE Leng & $1,380 * * *$ & $0,00795 * * *$ & & & & \\
\hline SIMCE Mat & & & $1,252 * * *$ & $0,00735^{* * * *}$ & & \\
\hline Constante & $134,6 * * *$ & $-2,163 * * *$ & $157,0 * * *$ & $-1,960 * * *$ & $84,75 * * *$ & $-2,590 * * *$ \\
\hline $\mathrm{N}^{\mathrm{o}}$ observaciones & 225265 & 225265 & 225265 & 225265 & 225265 & 225265 \\
\hline
\end{tabular}

Fuente: elaboración propia sobre la base de datos del Sistema de Medición de Calidad de la Educación (SIMCE) 2006 y la Prueba de Selección Universitaria (PSU) 2008.

Nota: $* * * \mathrm{p}<0,01, * * \mathrm{p}<0,05, * \mathrm{p}<0,1$.

SIMCE Leng: SIMCE Lenguaje.

SIMCE Mat: SIMCE Matemáticas.

NSE: nivel socioeconómico.

\section{Bibliografía}

Arrow, K., S. Bowles y S. Durlauf (eds.) (2000), Meritocracy and Economic Inequality, Princeton, Princeton University Press.

Bourdieu, P. y J.C. Passeron (1964), Los herederos. Los estudiantes y la cultura, Buenos Aires, Siglo Veintiuno Editores.

Brunner, J.J. y G. Elacqua (2003), "Factores que inciden en una educación efectiva. Evidencia internacional", La educación: Revista Interamericana de Desarrollo Educativo, $\mathrm{N}^{\circ} 139$ 140 (I-II), Washington, D.C., Organización de los Estados Americanos (OEA).

CEPAL (Comisión Económica para América Latina y el Caribe) (2010), La hora de la igualdad. Brechas por cerrar, caminos por abrir (LC/G.2432(SES.33/3)), Santiago de Chile.

Chumacero, R., D. Gómez Caorsi y R. Paredes (2008), "I Would Walk 500 Miles (If it Paid)" [en línea] http://mpra.ub.uni-muenchen. de/15125/

CRUCH (Consejo de Rectores de las Universidades Chilenas) (2012) [en línea] http://www.consejoderectores.cl/web/

Contreras, D. (1999), "Distribución del ingreso en Chile. Nueve hechos y algunos mitos", Perspectivas, vol. 2, No 2, Santiago de Chile, Universidad de Chile.

(1996), Pobreza y desigualdad en Chile: 1987-1992. Discurso, metodología y evidencia empírica, Santiago de Chile, Centro de Estudios Públicos (CEP).

Contreras, D. y S. Gallegos (2011), "Desigualdad salarial en América Latina: Una década de cambios", Revista de la CEPAL, $\mathrm{N}^{\circ} 103$ (LC/G.2487-P), Santiago de Chile.

Contreras, D., S. Gallegos y F. Meneses (2009), Determinantes de desempeño universitario. ¿Importa la habilidad relativa?, Santiago de Chile, Consejo Nacional de Educación.
Contreras, D. y V. Macías (2002), "Desigualdad educacional en Chile: Geografía y dependencia”, Cuadernos de economía, vol. 39, $\mathrm{N}^{\mathrm{o}}$ 118, Santiago de Chile, Instituto de Economía, Pontificia Universidad Católica de Chile.

Contreras, D., P. Sepúlveda y S. Bustos (2007), "When schools are the ones that choose: the effects of screening in Chile", Documentos de trabajo, $\mathrm{N}^{\circ} 242$, Santiago de Chile, Universidad de Chile.

Cornejo, R. (2005), "Variables y factores asociados al aprendizaje escolar. Una discusión desde la investigación actual”, Santiago de Chile, Universidad de Chile.

DEMRE (Departamento de Evaluación, Medición y Registro Educacional) (2012), "La Prueba de Selección Universitaria", Santiago de Chile [en línea] www.demre.cl/psu.htm>

Elacqua, G. y R. Fábrega (2004), El consumidor de la educación: El actor olvidado de la libre elección de escuelas en Chile, Santiago de Chile.

Elaqcua, G., S. González y F. Salazar (s/f), "Educación superior: acceso, permanencia y perfil social de los graduados comparados con los egresados de la educación media. Apuntes sobre el caso chileno", Sistema de Información de Tendencias Educativas en América Latina (SITEAL).

Epple, D. y R. Romano (1998), "Competition between private and public schools, vouchers and peer group effects", American Economic Review, vol. 88, № 1, Nashville, Tennessee, American Economic Association.

Gallego, F. y A. Hernando (2009), "School choice in Chile: looking at the demand side", Documentos de trabajo, N $\mathrm{N}^{\circ} 356$, Santiago de Chile, Instituto de Economía, Pontificia Universidad Católica de Chile. 
González, P. (s/f), "Dos desafíos de equidad desde la economía a la educación", Santiago de Chile, Centro de Economía Aplicada de la Universidad de Chile/Fondo de las Naciones Unidas para la Infancia (UNICEF).

Hanushek, E. (1996), "Measuring investment in education", Journal of Economic Perspectives, vol. 10, No 4, Nashville, Tennessee, American Economic Association.

Hanushek, E. y L. Wößmann (2008), "Education and economic growth", capítulo preparado para la International Encyclopedia of Education.

Hanushek, E., S. Rivkin y L. Taylor (1996), "Aggregation and the estimated effects of school resources", The Review of Economics and Statistics, vol. 78, No 4, Cambridge, Massachusetts, The MIт Press.

Heckman, J. (1979), "Sample selection bias as a specification error", Econometrica, vol. 47, No 1, Nueva York, The Econometric Society.

Hsieh, C. y M. Urquiola (2006), "The effects of generalized school choice on achievement and stratification: evidence from Chile's voucher program", Journal of Public Economics, vol. 90, № 8-9, Amsterdam, Elsevier.

Krueger, A. (2000), 'An Economist's View' of Class Size Research, Santa Monica, Milken Institute.

López-Calva, L. y N. Lustig (2010), Declining Latin America Inequality: Market Forces or State Action?, Washington, D.C., Brookings Institution Press/Programa de las Naciones Unidas para el Desarrollo (PNUD).

McEwan, P. y M. Carnoy (1999), "The Effectiveness and Efficiency of Private Schools in Chile's Voucher System", Washington, D.C., Banco Mundial.
Mizala, A. y P. Romaguera (2000), "Determinación de factores explicativos de los resultados escolares en educación media en Chile", Serie Economía, № 85, Santiago de Chile, Centro de Economía Aplicada, Departamento de Ingeniería Industrial, Facultad de Ciencias Físicas y Matemáticas, Universidad de Chile.

Núñez, J. y C. Risco (2004), "Movilidad intergeneracional del ingreso en un país en desarrollo: El caso de Chile", Documentos de trabajo, $\mathrm{N}^{\circ} 210$, Santiago de Chile, Departamento de Economía, Universidad de Chile.

OCDE (Organización de Cooperación y Desarrollo Económicos) (2012), "Better Life Index" [en línea] http://www.oecdbetterlifeindex. org/topics/income/

(2011), Maintaining Momentum: OECD Perspectives on Policy Challenges in Chile, París.

(2007), Education at a Glace 2007: OECD Indicators, París.

Puhani, A. (2000), "The Heckman correction for sample selection and its critique", Journal of Economic Surveys, vol. 14, No 1 , Wiley Blackwell.

Todd, E. e I. Wolpin (2003), "On the specification and estimation of the production function for cognitive achievement", The Economic Journal, vol. 113, № 485, Royal Economic Society.

Valenzuela, J. (2006), Evolución de la segregación socioeconómica de los estudiantes chilenos y su relación con el financiamiento compartido, Santiago de Chile, Ministerio de Educación.

Williamson, J. (2003), An Agenda for Restarting Growth and Reform, Washington, D.C., Institute for International Economics. 